\title{
Development of a GC-FID method for the quantitative determination of polyglycerol polyricinoleate (PGPR) in foods
}

\author{
Chunxia Su, b, Paul Van der Meeren ${ }^{a}$, Bruno De Meulenaer ${ }^{b}$ \\ a Particle and Interfacial Technology Group, Department of Green Chemistry \& \\ Technology, Faculty of Bioscience Engineering, Ghent University, Coupure Links 653, \\ 9000 Ghent, Belgium \\ b NutriFOODchem Unit, Department of Food Technology, Safety and Health, Faculty of \\ Bioscience Engineering, Ghent University, Coupure Links 653, B-9000 Ghent, Belgium
}

The food additive polyglycerol polyricinoleate (PGPR, E 476) is a powerful lipophilic emulsifier used in low-fat spreads and chocolate. It is manufactured by esterification of condensed castor oil fatty acids with polyglycerol. PGPR typically consists of a complex mixture of various esters with a varying polymerisation degree (both on the level of glycerol and ricinoleic acid) and a varying esterification degree. It should be used at the lowest level at which the desired technological effect is achieved, not exceeding the specified maxima according to the Annex II to Regulation (EC) No 1333/2008. In order to enforce the specified legal restrictions, analytical methods should be available enabling to determine the actual PGPR content in foods. Because of the complex composition of commercial PGPR products, it is impossible to determine the individual PGPR species present and consider their sum as total PGPR content. Therefore, in this study, an indirect approach is developed based on the fact that ricinoleic acid constitutes approximately $90 \%$ of the fatty acid content of castor oil, whereas it is not present in food products as such. This method is based on the determination of the average ricinoleic acid content by using 12-hydroxyoctadecanoic acid as an internal standard, from which the PGPR concentration was deduced. The method involved saponification, methylation, a two-step solid phase extraction (SPE) separation of the fatty acid methyl esters (FAMEs), silylation, and gas chromatography-flame ionization detection (GC-FID) analysis. The results obtained showed that the average content of ricinoleic acid in a representative set of commercial PGPR samples was $86.63 \pm 1.98 \mathrm{~g} / 100 \mathrm{~g}$ sample. Further, the developed method was validated by determining the PGPR recovery from a high oleic sunflower oil (HOSO) solution, chocolate spiked with a commercially available PGPR, and commercially available low-fat margarines with a known PGPR content. The recovery was more than $95 \%$ for all matrices, indicating the accuracy of the developed analytical technique. Moreover the method proved to be very reproducible $(\mathrm{RSD}<4$ $\%$ for concentrations ranging between 0.2 to $5 \mathrm{wt} \%)$. The results indicated that our proposed GC-FID method enabled the quantitative determination of the PGPR concentration in commercial food products with various fat contents in a reliable way. 